\title{
IFN- $\gamma$ response and IL-8 plasma levels in neonates with respiratory syncytial virus bronchiolitis
}

\section{To the Editors:}

In a recent article published in the European Respiratory Journal, BonT et al. [1] presented data on systemic cytokine responses in hospitalized ventilated and nonventilated infants with respiratory syncytial virus
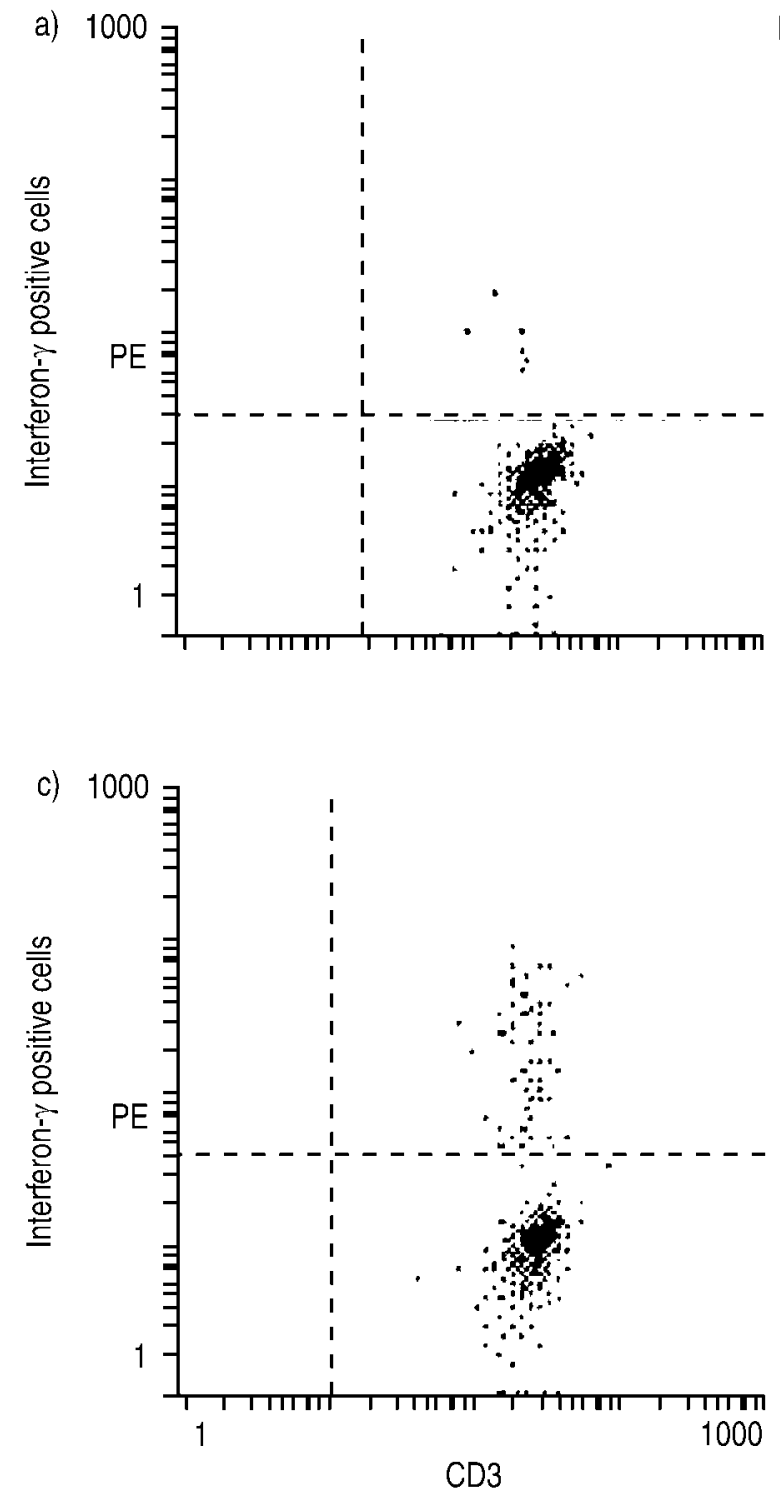

(RSV) bronchiolitis. Their main findings were a lower production of interferon- $\gamma($ IFN- $\gamma)$ and elevated plasma levels of interleukin (IL)-8 in ventilated patients. They concluded from their data that a depressed lymphocyte function and elevated IL-8 plasma levels are markers of severe RSV bronchiolitis in infants requiring ventilation.

b)

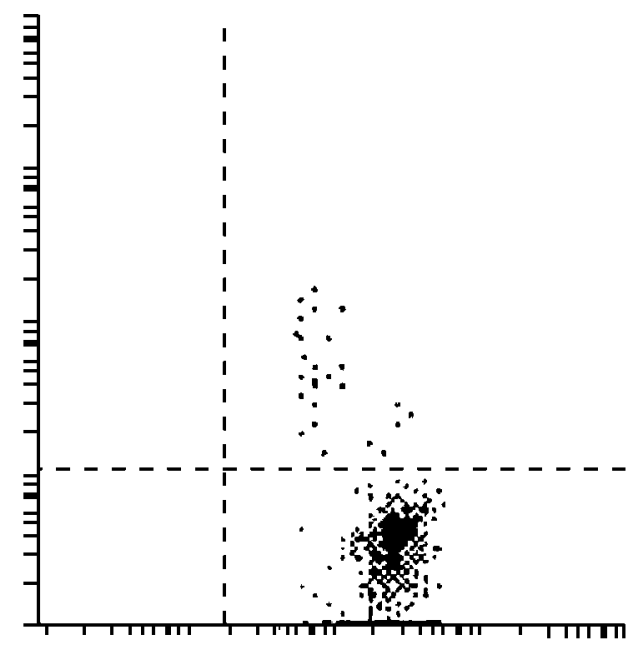

d)

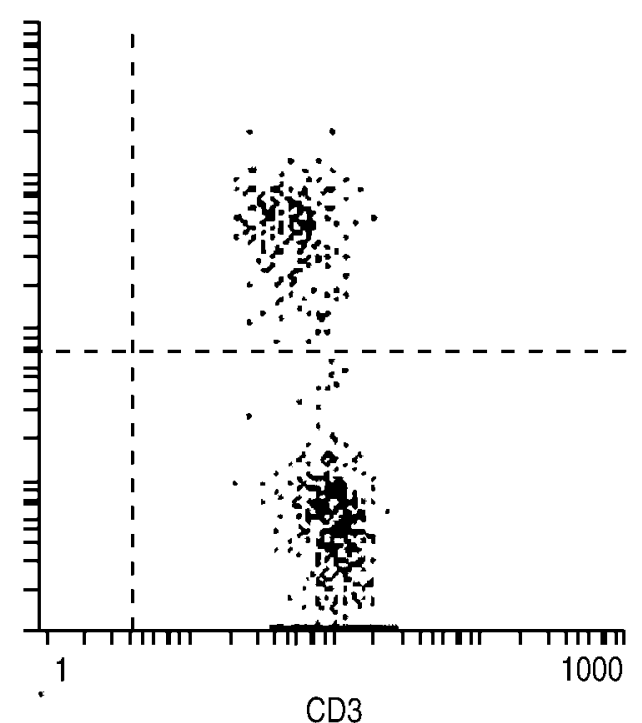

Fig. 1. - Flow cytometric detection of intracytoplasmic interferon (IFN)- $\gamma$, synthesis among CD3 positive lymphocytes stimulated with phorbol 12-myristate 13-acetate and ionomycin after cell permeabilization with saponin. Representative cases illustrate the age dependent IFN- $\gamma$ synthesis in lymphocytes. Only small amounts of IFN- $\gamma$ producing lymphocytes were detectable in a) healthy preterm and b) term infants at birth. In an c) infant 4 months of age the number of IFN- $\gamma$ producing lymphocytes increased and reached $40 \%$ of an d) adult. The right upper quadrant represents the per cent IFN- $\gamma$ producing lymphocytes of the total CD3 positive lymphocytes population: preterm infant: $2 \%$; term infant: $6 \%$; infant, 4 months: $14 \%$; adult: $34 \%$. Percentages in the right lower quadrant represent the cytokine negative lymphocytes.

ERJ (RJ73284_16_Live.3d) 13/4/01 10:58:41 Rev 6.06e/W (Aug 31 2000) 


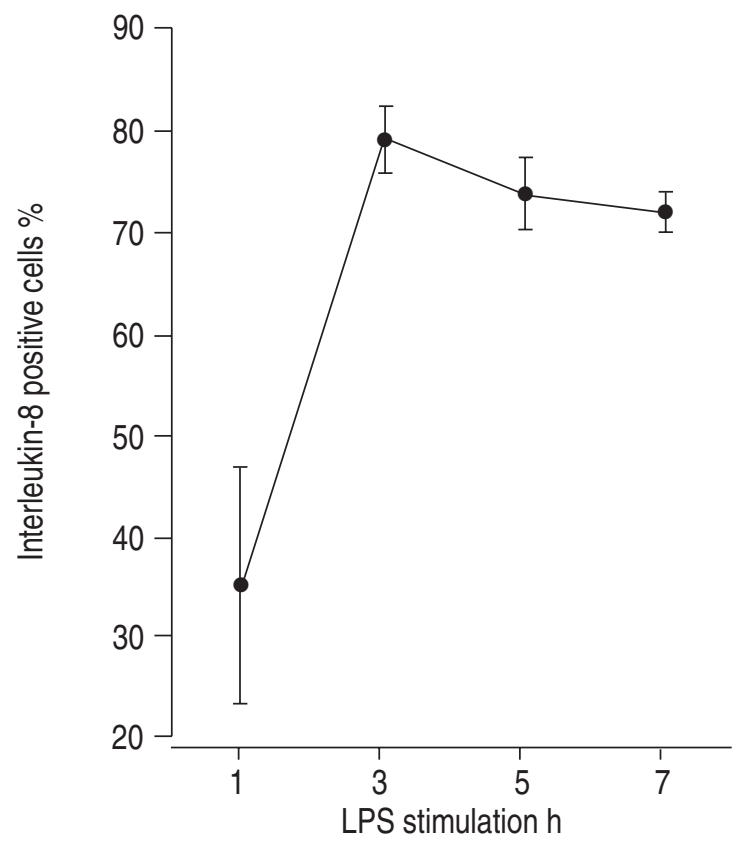

Fig. 2. - Kinetics of intracytoplasmic interleukin (IL)-8 synthesis in monocytes $(n=5)$. IL- 8 producing monocytes were already detectable $1 \mathrm{~h}$ after stimulation with lipopolysaccharide (LPS) in whole blood culture followed by a peak after $3 \mathrm{~h}$. The amount of IL-8 producing monocytes is expressed as the percentage of the CD14 positive monocyte population analysed by flow cytometry at the single cell level according to a modified protocol [3]. Bars refer to 1 SEM.

However, the median age in ventilated patients was significantly lower than in nonventilated patients $(1$ versus 4 months). Furthermore, eight out of 14 ventilated patients were prematurely born. From that point of view it is difficult to compare these different groups because IFN- $\gamma$ response is age-related and increases after 2 months of age [2]. Performing a recently developed technique we were able to investigate IFN- $\gamma$ production in neonatal and adult lymphocytes directly at the single cell level by flow cytometry [3]. The number of IFN- $\gamma$ producing lymphocytes was strikingly lower in newborn and especially preterm infants, followed by a substantial increase in the first months of life (fig. 1). Considering these data, the lower IFN- $\gamma$ response in the group of ventilated infants was more age-dependent than disease-related. Additionally, it seems questionable whether a local RSV infection of the lung is able to suppress IFN- $\gamma$ production in blood lymphocytes supraphysiologically stimulated with phytohaemagglutinin.

The elevated IL-8 plasma levels described in ventilated infants as a marker of severe RSV bronchiolitis also needs further comment [1]. Although the difference in IL-8 plasma levels between ventilated and nonventilated infants was reported to be significant it seems questionable whether this difference is clinically important. Indeed, ten out of 14 cytokine levels from ventilated infants fell in the range of nonventilated infants [1]. In addition, in an experimental rat model it has been demonstrated that mechanical ventilation can stimulate local and systemic inflammatory reactions [4].
Furthermore, IL- 8 levels were found to be elevated in ventilated preterm infants who develop chronic lung disease $[5,6]$. These studies support the view that mechanical ventilation induces inflammation in newborn infants and could be responsible for elevated IL-8 plasma levels in the group of ventilated infants with RSV bronchiolitis, also [1]. BonT et al. [1] argued that a period of 2-6 h between initiation of mechanical ventilation and blood sampling was too short for induction of inflammation. However, we could demonstrate a fast IL-8 synthesis in monocytes ex vivo after $1 \mathrm{~h}$ of stimulation followed by a peak after $3 \mathrm{~h}$ (fig. 2). Thus, the influence of mechanical ventilation on IL-8 production in the group of ventilated infants with RSV bronchiolitis [1] cannot be excluded.

Considering these data, we think that neither interferon- $\gamma$ response or interleukin-8 plasma levels can be regarded as a marker of disease severity in respiratory syncytial virus bronchiolitis. It seems more likely that immune response maturation and mechanical ventilation were responsible for the differences between ventilated and nonventilated infants with respiratory syncytial virus bronchiolitis reported in the study by BonT et al. [1].

\section{Schultz, N. Richter, J.C. Möller, P. Bucsky}

Dept of Paediatrics, Medical Unversity of Lübeck, Ratzeburger Allee 160, 23538 Lübeck, Germany. Fax: 494515003767.

\section{References}

1. Bont L, Heijnen CJ, Kavelaars A, et al. Peripheral blood cytokine responses and disease severity in respiratory syncytial virus bronchiolitis. Eur Respir $J$ 1999; 14: $144-149$.

2. Frenkel L, Bryson YJ. Ontogeny of phytohemagglutinin-induced gamma interferon by leukocytes of healthy infants and children: evidence for decreased production in infants younger than 2 months of age. J Pediatr 1987; 111: 97-100.

3. Schultz C, Reiss I, Rott C, Richter N, Gortner L, Bucsky P. Intracytoplasmic detection of cytokines in neonatal lymphocytes and monocytes by flow cytometry. Blood 1999; 93: 3566-3567.

4. Tremblay L, Valenza F, Ribeiro SP, Li J, Slutsky AS. Injurious ventilatory strategies increases cytokines and c-fos mRNA expression in an isolated rat model. J Clin Invest 1997; 99: $944-952$.

5. McColm JR, McIntosh N. Interleukin-8 in bronchoalveolar lavage samples as predictor of chronic lung disease in premature infants. Lancet 1994; 343: 729 .

6. Groneck P, Goetze-Speer B, Oppermann M, Eiffert H, Speer CP. Association of pulmonary inflammation and increased microvascular permeability during the development of bronchopulmonary dysplasia: a sequential analysis of inflammatory mediators in respiratory fluids of high-risk preterm neonates. Pediatrics 1994; 93: 712-718. 


\section{From the authors:}

We read with interest the valuable comments by Schultz and colleagues to our article [1].

In our paper we showed that interferon (IFN)- $\gamma$ responses to nonspecific stimuli in whole blood cultures are lower in mechanically ventilated infants than in hospitalized nonventilated infants during the acute phase of respiratory syncytial virus (RSV) bronchiolitis. Schultz and colleagues argue that this difference might be caused by the age in mechanically ventilated infants, which is significantly lower than in nonventilated infants. They illustrate this by showing that neonates have lower numbers of IFN- $\gamma$ producing cells than older infants.

We investigated the relation between age and cytokine responses and between age and lymphoproliferative responses in a group of healthy controls, including three premature neonates. Indeed, IFN- $\gamma$ responses correlated with age (fig. 1). However, practically the complete range of IFN- $\gamma$ responses in ventilated patients is below the minimum IFN- $\gamma$ response in nonventilated patients and control infants, strongly suggesting that age is not an important determining factor explaining the differences we found in IFN- $\gamma$ responses.

To further investigate whether decreased IFN- $\gamma$ responses are explained by immaturity of the immune system, we investigated cytokine responses over time in a separate group of eight mechanically ventilated RSV bronchiolitis patients. In figure 2 , it is shown that IFN$\gamma$ responses increased rapidly during the first $1-2$ weeks after initiation of mechanical ventilation. We therefore, conclude that decreased IFN- $\gamma$ responses in mechanically ventilated RSV bronchiolitis patients is not the result of an immature immune system.

Finally, we note that interleukin (IL)-4 responses and lymphoproliferative responses were also lower in mechanically ventilated infants than in hospitalized RSV patients, which is not explained by age (figs. 3 and 4).

In the paper we showed that plasma IL-8 levels are higher in mechanically ventilated patients than in nonventilated patients during the acute phase of RSV

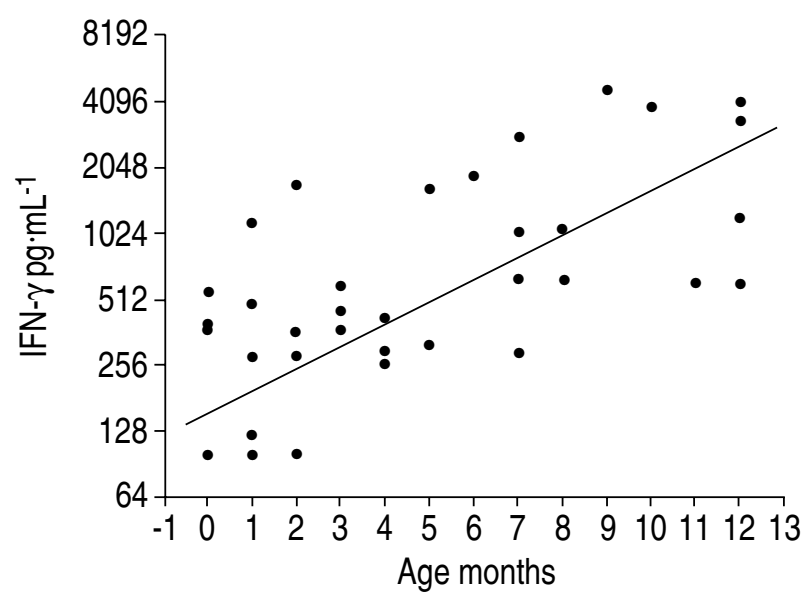

Fig. 1. - Relation between age and interferon (IFN) $-\gamma$ responses in healthy infants.

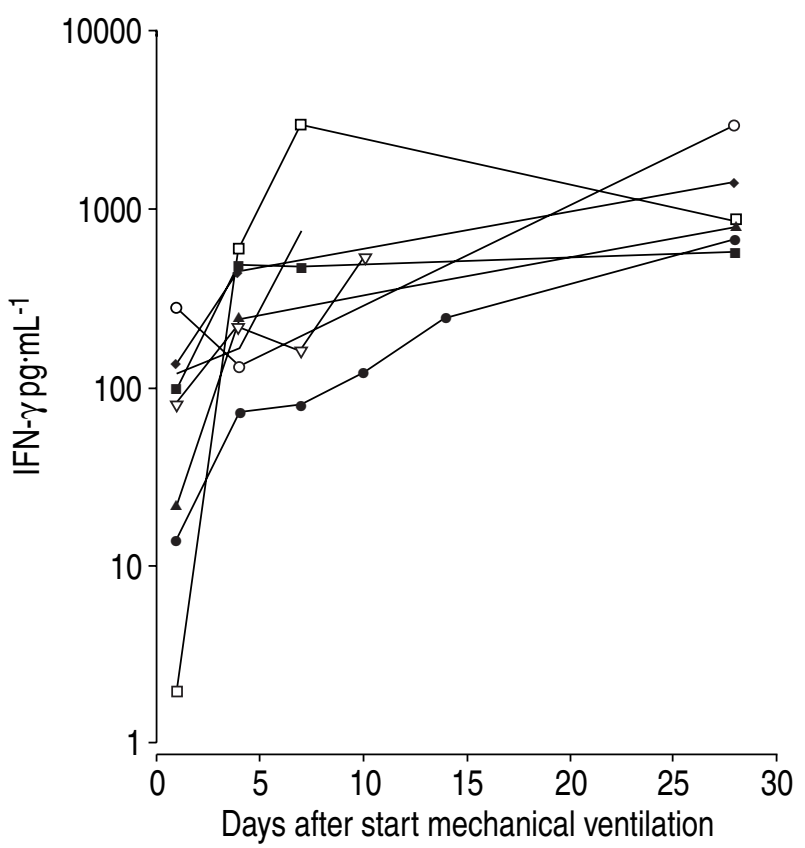

Fig. 2. - Interferon (IFN) $-\gamma$ responses over time in eight mechanically ventilated respiratory syncytial virus bronchiolitis patients. Samples were taken at initiation of mechanical ventilation and subsequently, every $72 \mathrm{~h}$. Finally a sample was taken 3 weeks after initiation of mechanical ventilation.

bronchiolitis. Schultz and colleagues are concerned that elevated plasma IL-8 found in mechanically ventilated patients are the result of mechanical ventilation and not of disease severity. This is illustrated by showing that IL-8 producing monocytes can be demonstrated after $1-3 \mathrm{~h}$ following initiation of mechanical ventilation in their model.

In our opinion it is difficult to determine the relevance of the percentage of monocytes that are positive for intracellular interleukin-8 after lipopolysaccharide stimulation for interleukin-8 plasma concentrations during mechanical ventilation during respiratory syncytial virus infection. First, monocytes are not the only

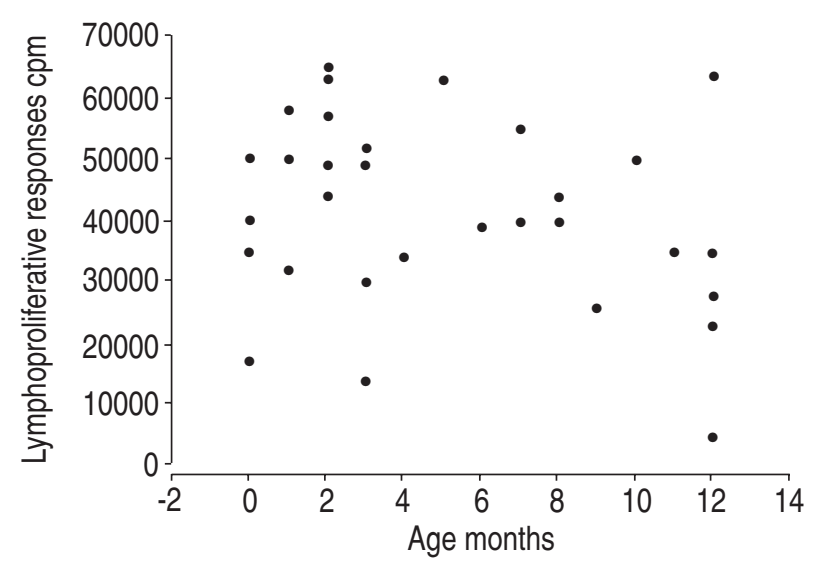

Fig. 3. - Relation between age and lymphoproliferative responses in healthy infants. 


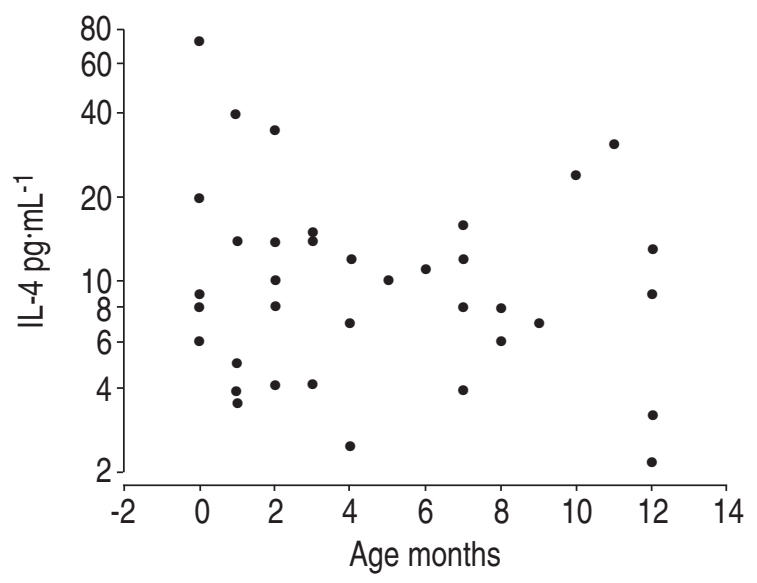

Fig. 4. - Relation between age and interleukin (IL) - 4 responses in healthy infants.

source of interleukin-8 and it is likely that epithelial cells also considerably contribute to interleukin-8 levels in vivo. Second, intracellular interleukin-8 is not yet secreted in the plasma. However, we agree with Schultz that "we cannot exclude a possible influence of mechanical ventilation on the immune responses found in ventilated patients in our study" as we already stated in the article.

We hope we have addressed all issues adequately.

\section{Bont, J.L.L. Kimpen}

Wilhelmina Children's Hospital, University Medical Centre Utrecht, POB 85090, NL 3508 AB Utrecht, The Netherlands. Fax: 31302505346

\section{References}

1. Bont L, Heijnen CJ, Kavelaars A, et al. Peripheral blood cytokine responses and disease severity in respiratory syncytial virus bronchiolitis. Eur Respir $J$ 1999; 14: $144-149$ 